\title{
A Test and Evaluation Method for Smart Fully Mechanized Mining Robot Production System
}

Jiacheng Xie ( $\nabla$ tyutxiejiacheng@qq.com )

Taiyuan University of Technology

\section{Fuxiang Ge}

Taiyuan University of Technology

\section{Tao Cui}

Taiyuan University of Technology

\section{Xuewen Wang}

Taiyuan University of Technology

\section{Research}

Keywords: Smart mining, Mining robot, Digital twin, Virtual simulation, Test and evaluation

Posted Date: May 5th, 2021

DOl: https://doi.org/10.21203/rs.3.rs-480264/v1

License: (c) (i) This work is licensed under a Creative Commons Attribution 4.0 International License. Read Full License 


\section{Abstract}

A test and evaluation method for smart fully mechanized mining robot production system is proposed. Based on the actual operation data of the geology and equipment of a particular working face, the kinematic models between equipment and coal seam are established. The virtual off-line operation system of fully mechanized coal mining face is constructed. The mining situation of virtual operation of working face reproduced and the simulation initial data and virtual scene operation data are determined. The perception operation model is added to the virtual equipment and Al robot analysis system is constructed. Based on sensor error analysis, execution error analysis and other error analysis, the equipment and geological exploration means are input according to the parameters of smart development and operation in the future. The operation evaluation system of fully mechanized coal mining face which considers cutting track, straightness, working space and dynamic coal seam is constructed. The operation of fully mechanized mining robot in the future is simulated, the development trend is determined and the robot operation performance is tested. The related prototype system is developed, and the testing of the overall operation of the working face was improved from the aspects of smart equipment and digital sensing elements. It shows that this method realizes the reappearance of fully mechanized mining operation process based on actual operation data and operation parameters. The current level of smart mining and some local or a small aspect of technological progress on the overall operation of the working face are analyzed and evaluated. The test and evaluation method points out the direction for the development of coal mine robot and smart mining.

\section{Full Text}

Due to technical limitations, full-text HTML conversion of this manuscript could not be completed. However, the manuscript can be downloaded and accessed as a PDF.

\section{Figures}




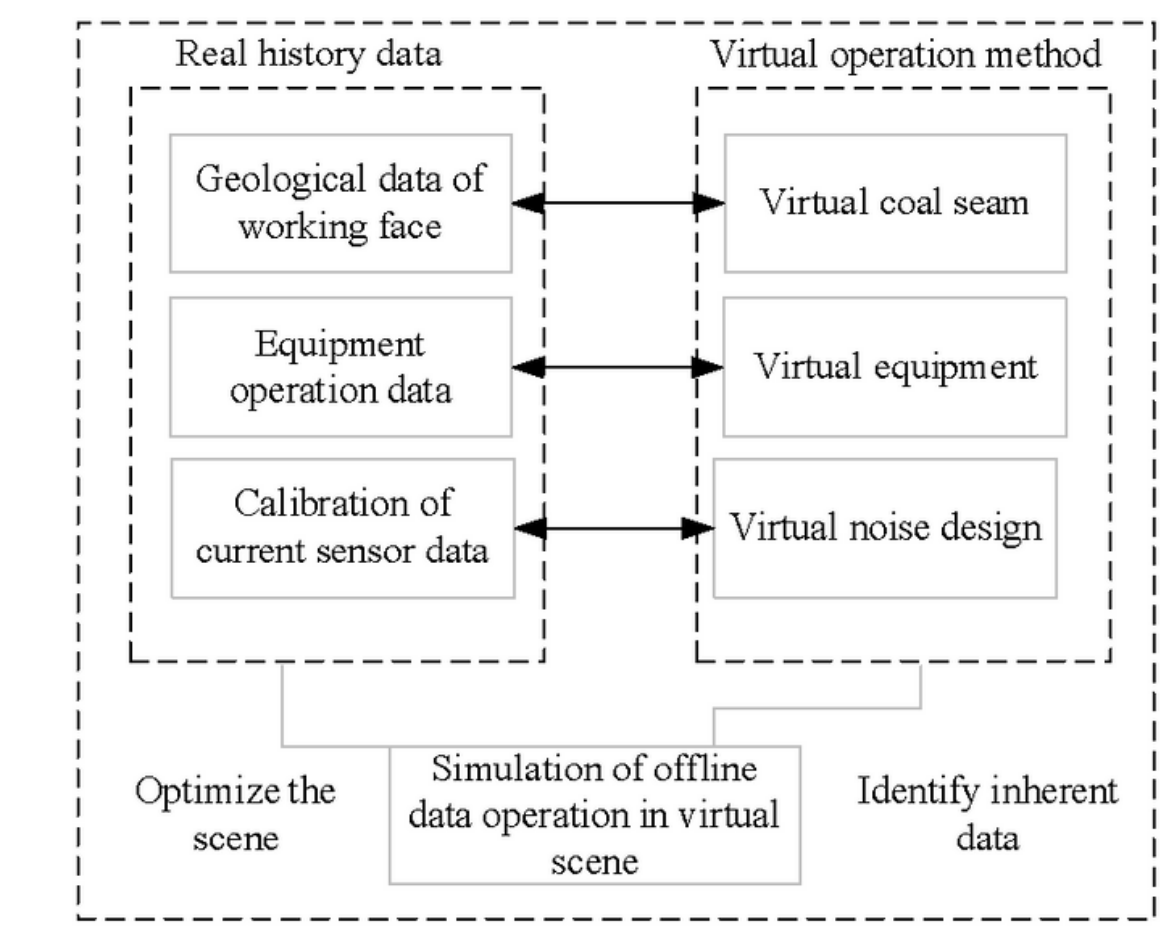

Virtual robot cooperative operation simulation system
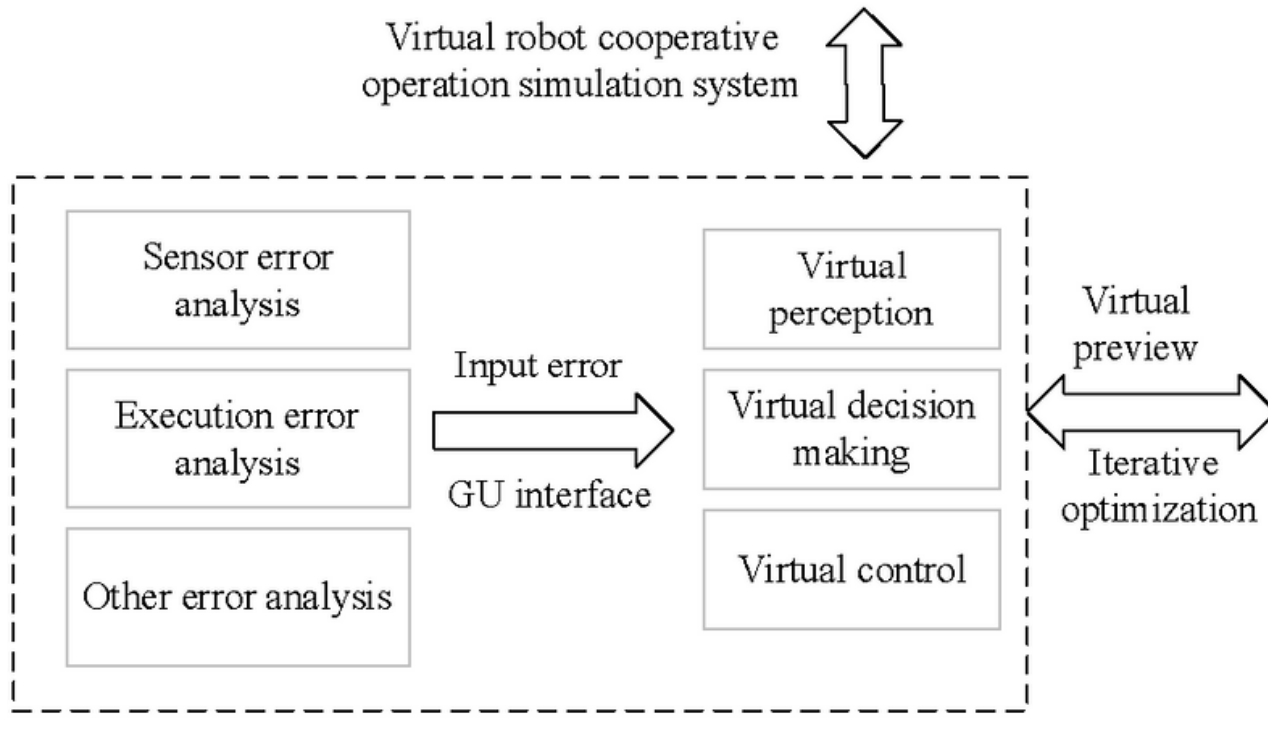

Evaluation system and index

Cutting trajectory prediction

Straightness of working face

Real time operation data monitoring system

\section{Figure 1}

Overall framework of test and evaluation method 


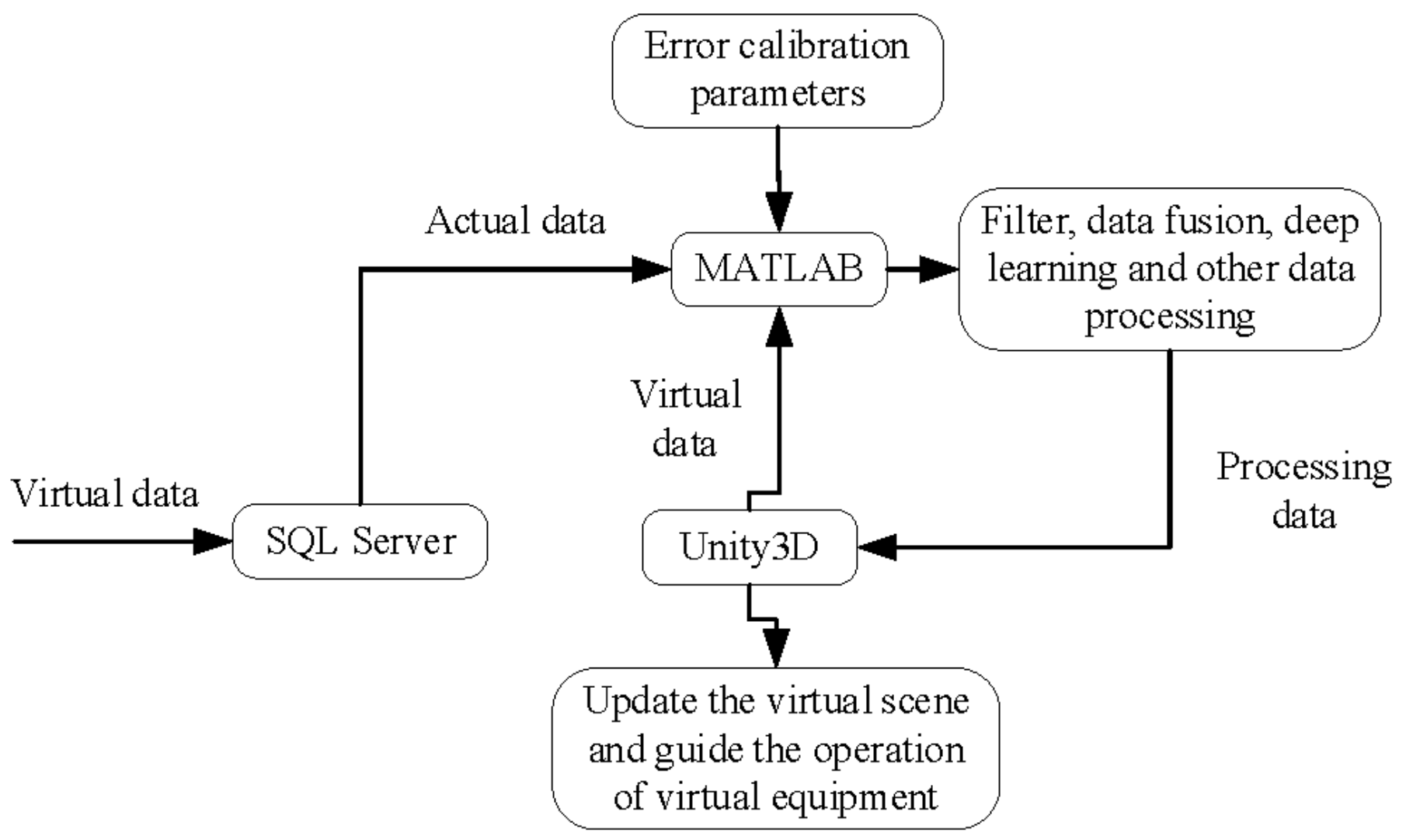

Figure 2

System software interaction

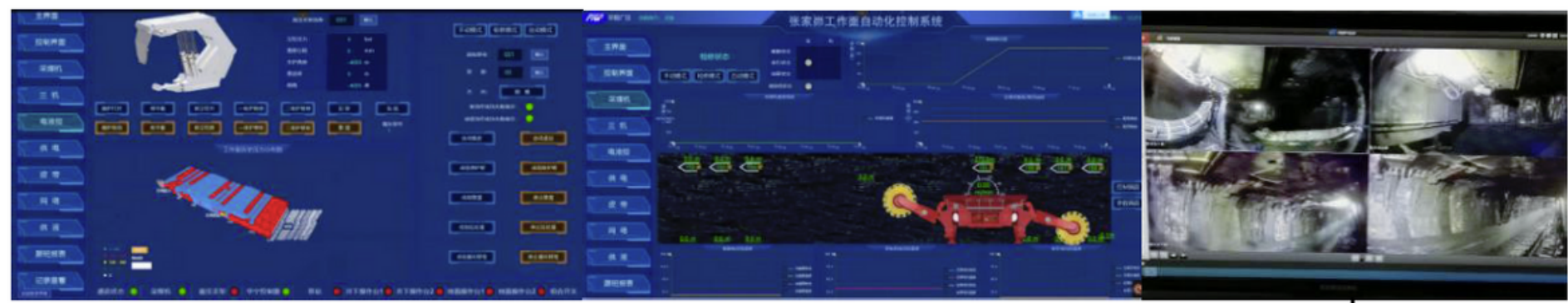

(a)Support monitoring interface

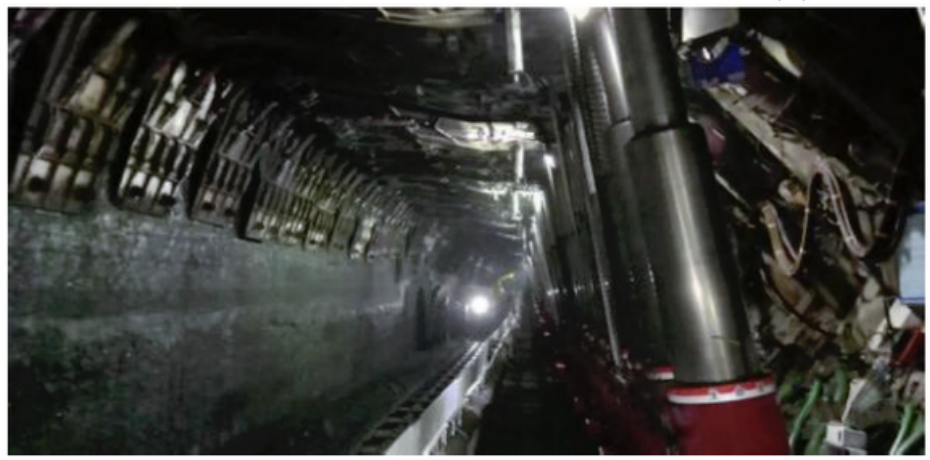

(d)Real scene of working face (b) Shearer monitoring interface
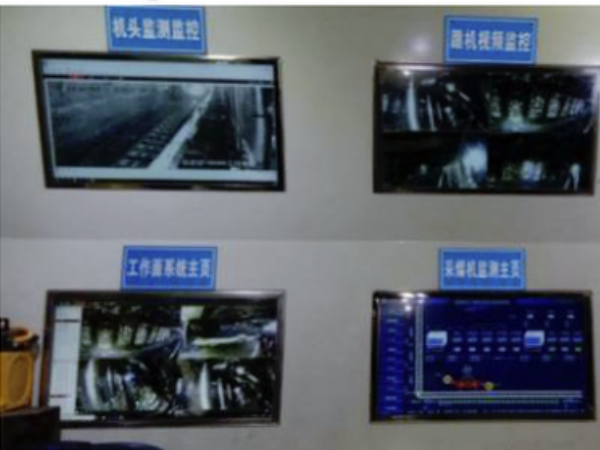

(c)Video surveillance

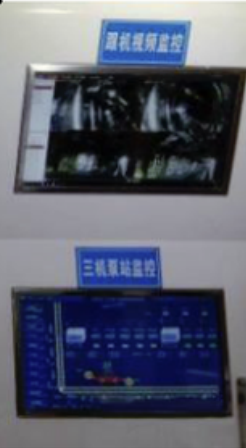

(e)Central control center

Figure 3 


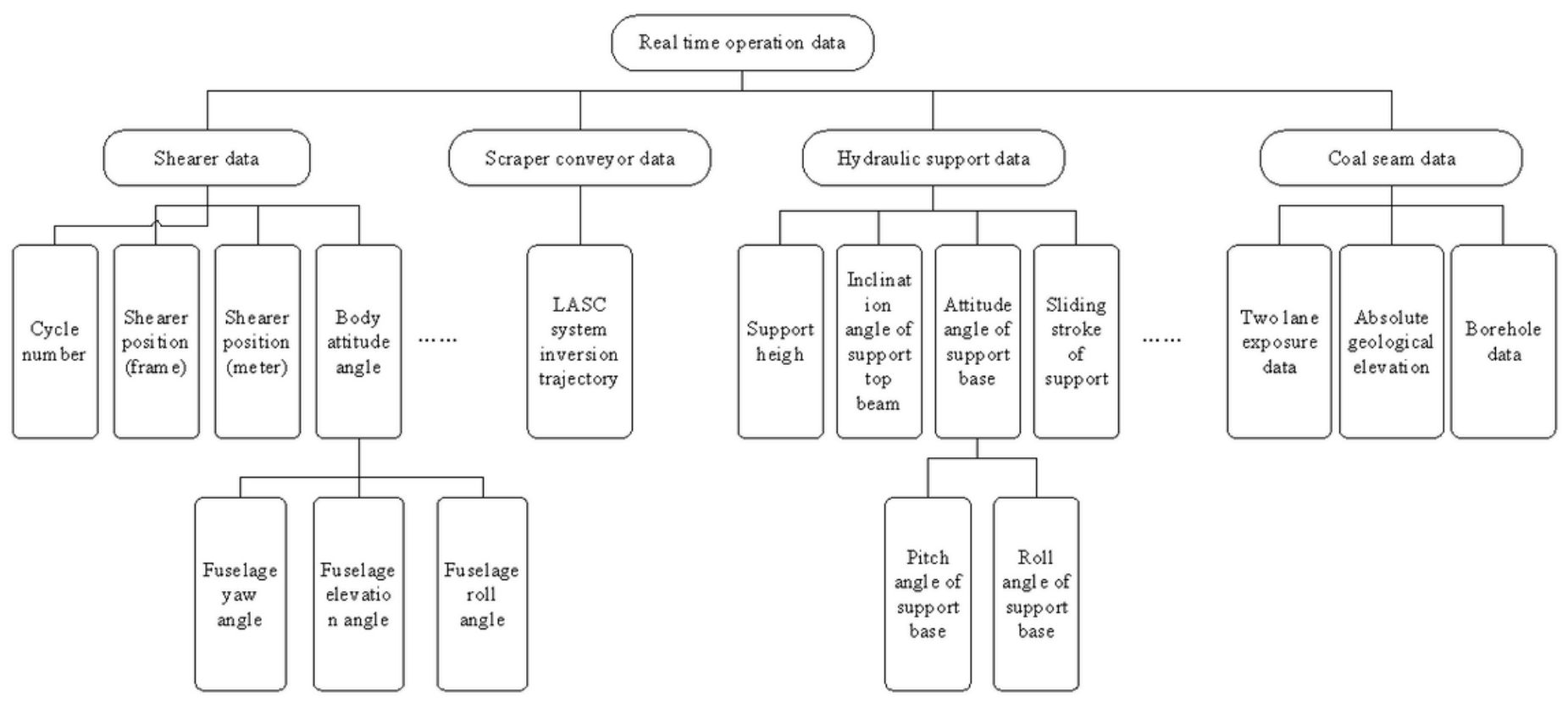

\section{Figure 4}

\section{Relevant equipment data and coal seam data of 100 cycles}

\begin{tabular}{|c|c|c|c|c|}
\hline \multicolumn{5}{|l|}{ Perceptual elemert } \\
\hline 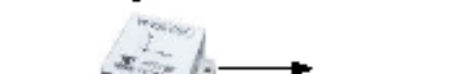 & $\begin{array}{l}\text { Extact virtual } \\
\text { angle variable }\end{array}$ & +Hoise & Delay & Current accuracy \\
\hline Tilts & $\begin{array}{l}\text { Extract virtual } \\
\text { angle variable }\end{array}$ & +Hoise & $50 \mathrm{~ns}$ & 0.3 degree \\
\hline Travel sersor & Extact virtual tavel varible & +Hoise & $50 \mathrm{~ms}$ & $50 m+50 m m$ \\
\hline Inertial navigation system Script & $\begin{array}{l}\text { Extact position } \\
\text { and attitude }\end{array}$ & +Hoise & $\begin{array}{c}500 \mathrm{~s} \\
20000 s^{+}\end{array}$ & $\begin{array}{l}\text { 3D angle } 0.1 \text { degress } \\
\text { Position enorl0nm }\end{array}$ \\
\hline $\begin{array}{c}\text { Coal and rock identification interaction, } \\
\text { method virtual }\end{array}$ & $\begin{array}{l}\text { Extract the irfomation } \\
\text { of coal seam }\end{array}$ & + Hoise & $\begin{array}{c}20005 \\
\text { is }\end{array}$ & $\mathrm{L} 0 \mathrm{~m}_{\mathrm{m}}$ \\
\hline $\begin{array}{l}\text { 3D modeling of coal seamin } \\
\text { geological explonation }\end{array}$ & $\begin{array}{l}\text { Dynamis coal seam } \\
\text { information }\end{array}$ & +Hoise & $\begin{array}{l}\text { Preset, } \\
\text { calculation per cycle }\end{array}$ & $>300 \mathrm{~mm}^{+}$ \\
\hline Camera & Virtual vision & +Hoise & $2 s$ & 50000 \\
\hline $\begin{array}{c}\text { Detection of ourvature and } \\
\text { staightress of scraper } \\
\text { correyor }\end{array}$ & Virtual detection & +Hoise & cabulation percycle & $\begin{array}{l}20 m m \\
40 m m\end{array}$ \\
\hline UAV detection scamring & Virtual detection & +Hoie & calculation per cycle & $>100 \mathrm{~mm}$ \\
\hline
\end{tabular}

\section{Figure 5}


Virtual offline operation system of fully mechanized working face

\begin{tabular}{|c|c|c|c|c|c|c|c|}
\hline \multicolumn{8}{|c|}{ Data optimization processing } \\
\hline $\begin{array}{l}\text { Shearer } \\
\text { positioning } \\
\text { module }\end{array}$ & \multicolumn{2}{|c|}{$\begin{array}{l}\text { Coupling } \\
\text { module of } \\
\text { shearer and } \\
\text { scraper } \\
\text { conveyor }\end{array}$} & \multicolumn{2}{|c|}{$\begin{array}{l}\text { Coupling } \\
\text { module of } \\
\text { scraper } \\
\text { conveyor and } \\
\text { hydraulic } \\
\text { support }\end{array}$} & \multicolumn{2}{|c|}{$\begin{array}{l}\text { Coupling } \\
\text { module of } \\
\text { cutting } \\
\text { trajectory and } \\
\text { roof and floor } \\
\text { of coal seam }\end{array}$} & $\begin{array}{l}\text { Cutting } \\
\text { trajectory } \\
\text { prediction } \\
\text { module }\end{array}$ \\
\hline \multicolumn{8}{|c|}{$\begin{array}{l}\text { Virtual operation monitoring system of } \\
\text { fully mechanized mining equipment }\end{array}$} \\
\hline $\begin{array}{l}\text { Constructio } \\
n \text { of } \\
\text { dynamic } \\
\text { coal seam }\end{array}$ & $\begin{array}{l}\text { Working } \\
\text { face shift }\end{array}$ & & & $\begin{array}{r}\text { Self st } \\
\text { hei } \\
\text { adjust } \\
\text { of sh }\end{array}$ & & $\begin{array}{c}\text { Coordinated } \\
\text { operation of } \\
\text { fully } \\
\text { mechanized } \\
\text { mining } \\
\text { equipment }\end{array}$ & $\begin{array}{c}\text { Equipment } \\
\text { operation } \\
\text { condition } \\
\text { monitoring }\end{array}$ \\
\hline
\end{tabular}

Figure 6

Off-line operation simulation system 


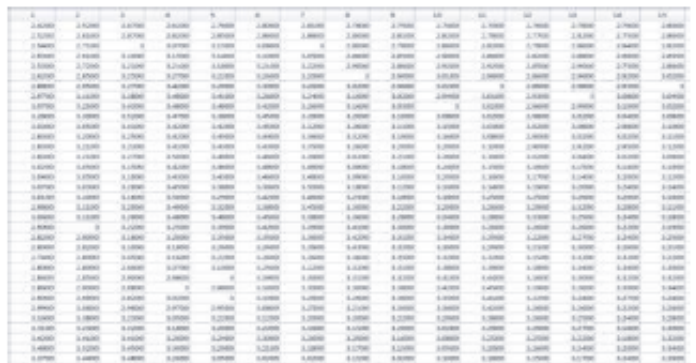

(a) Cutting data of shearer operation

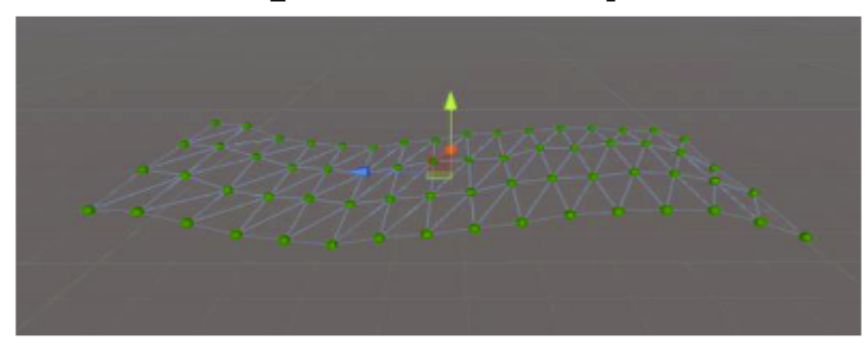

(c) Surface fitting

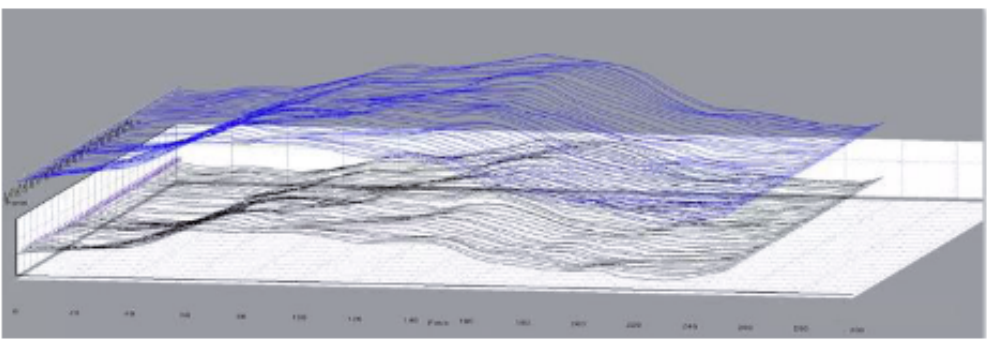

(b)Analvsis of cutting roof and floor model

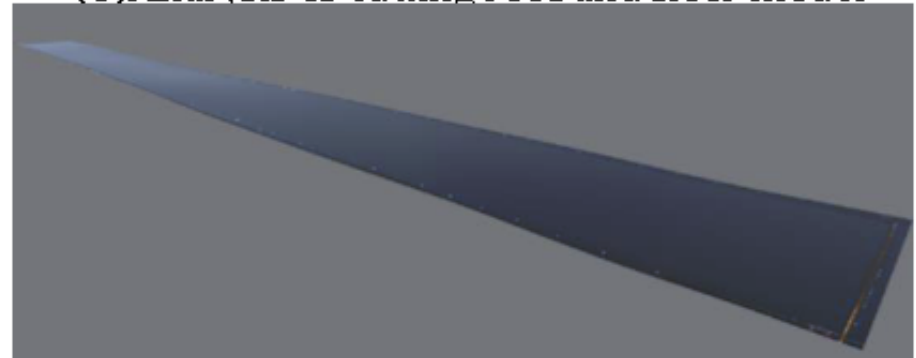

(d)Coal seam construction

Figure 7

Working face coordinate system 


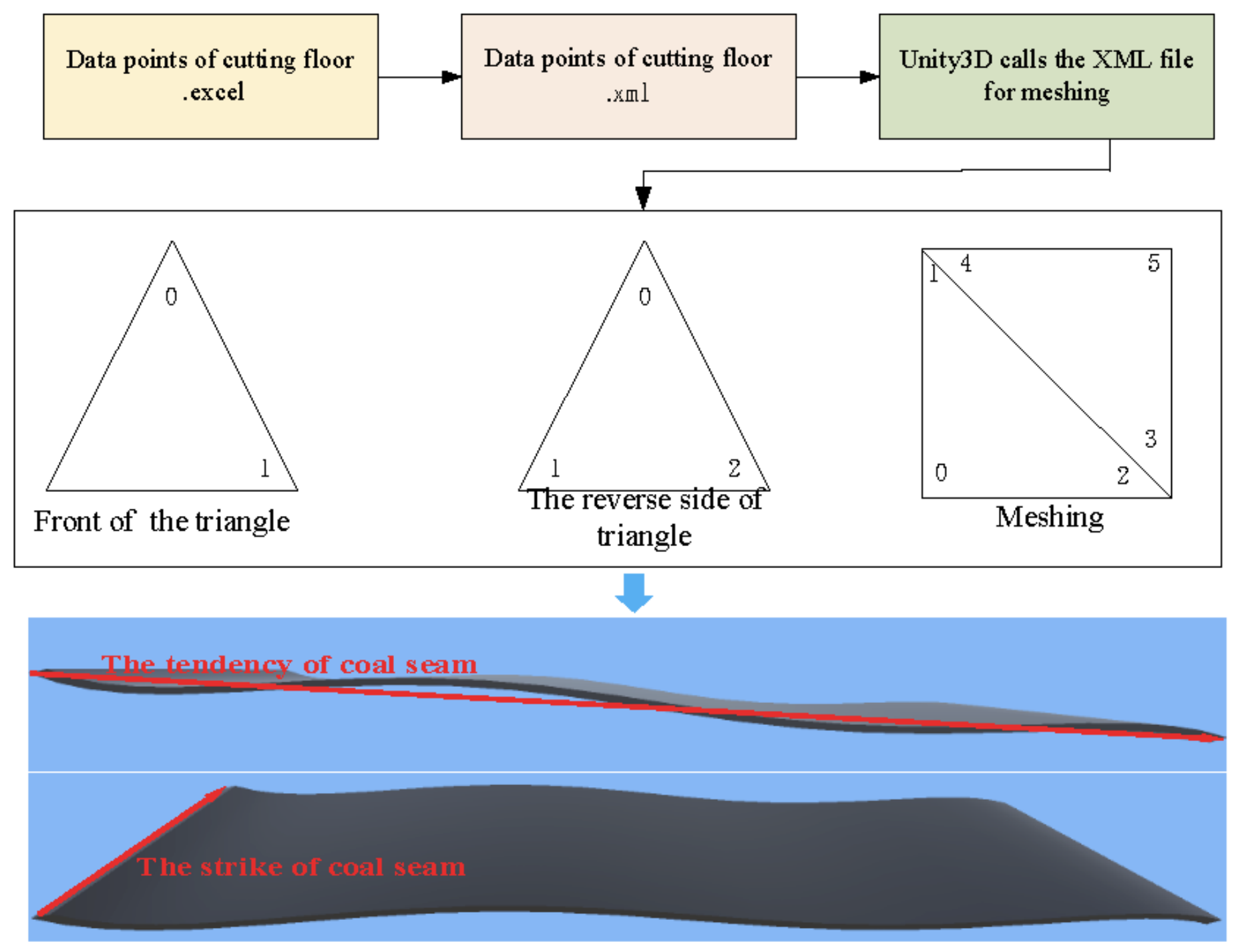

\section{Figure 8}

Construction method of coal seam cutting floor based on Mesh grid

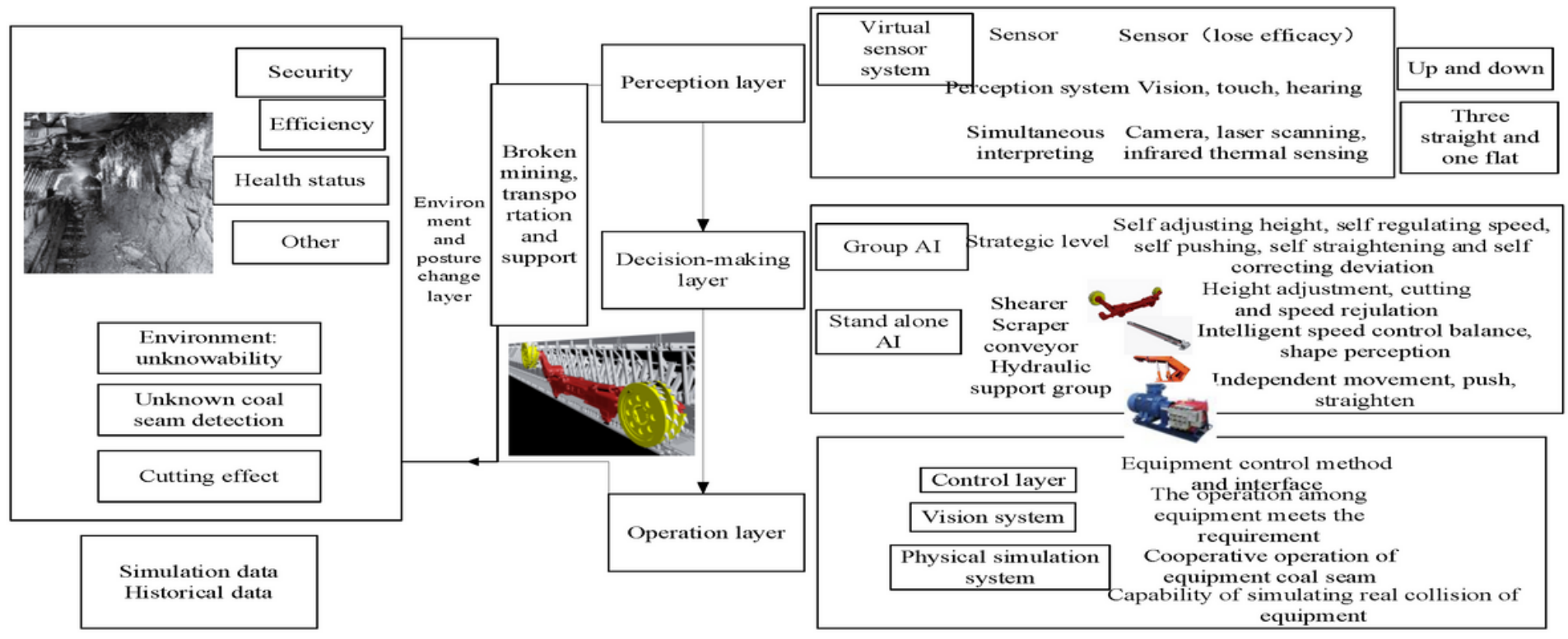




\section{Figure 9}

The general framework of Al operation of the virtual synthesis mining robot

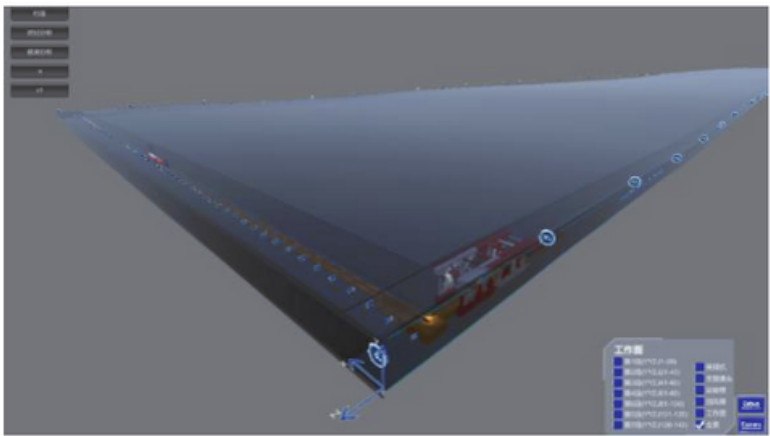

(a)Absolute coordinate system of virtual working face

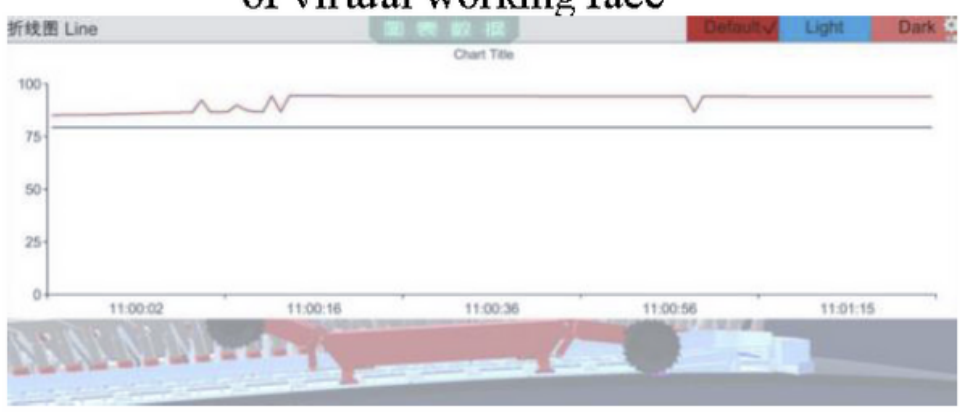

(c)Real time comparison of cutting curves

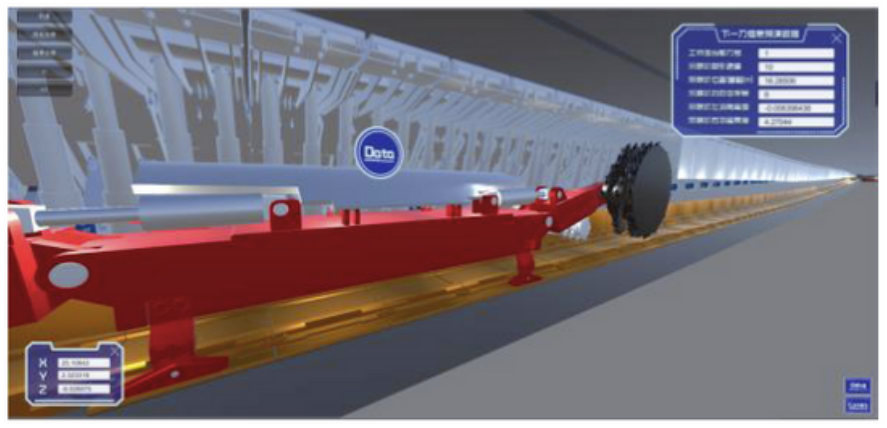

(b)Action reappearance of shearer

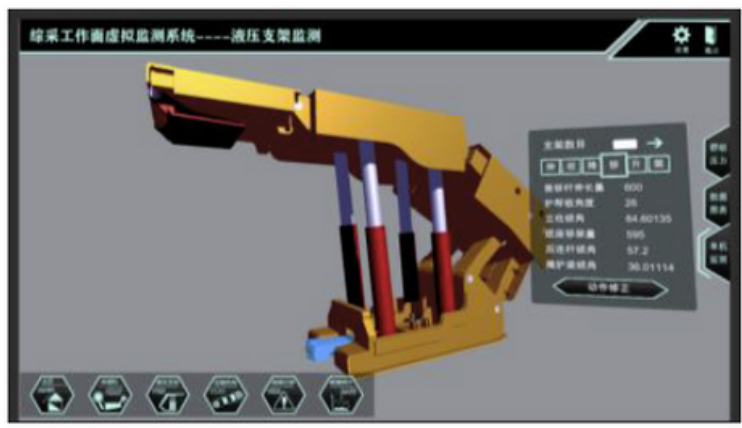

(d)Monitoring of single hydraulic support

Figure 10

Initial scenario construction and offline simulation results
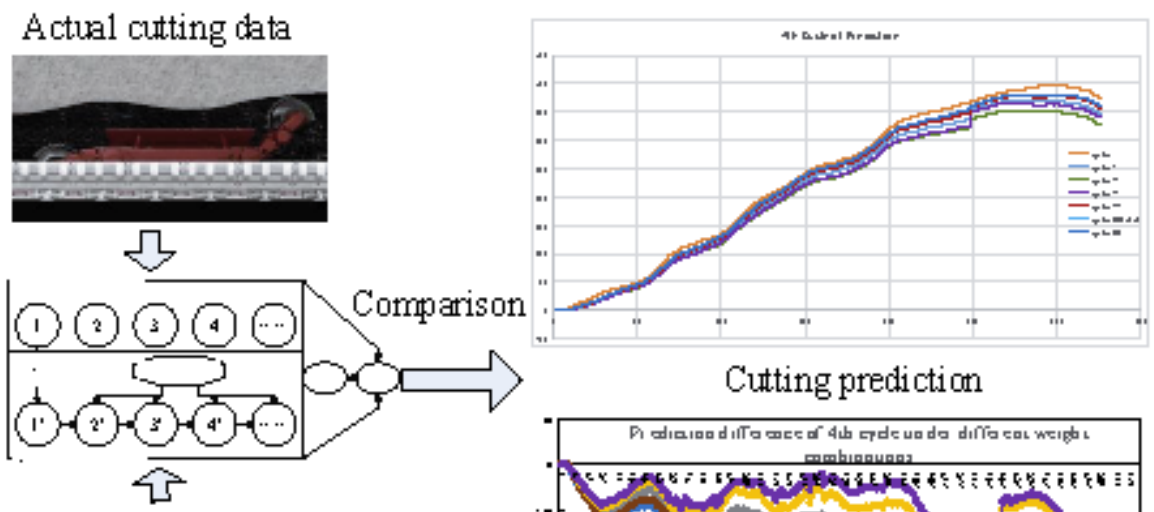

Cutting prediction

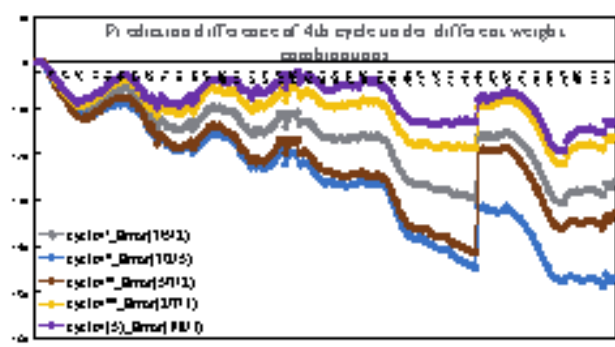

Error analysis of cutting pretiction

Figure 11 
Forecast coal seam

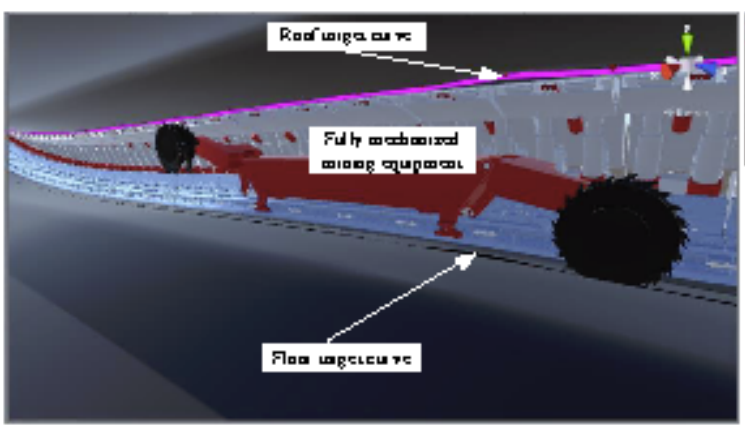

(a) Virtual equiment operation

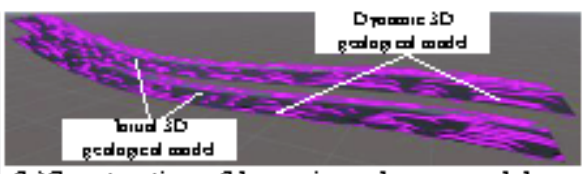

(b) Construction of dynamic coal seam model

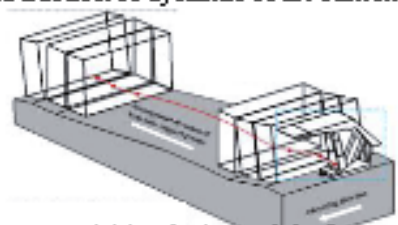

(c)Analysis model of working face straizhtress

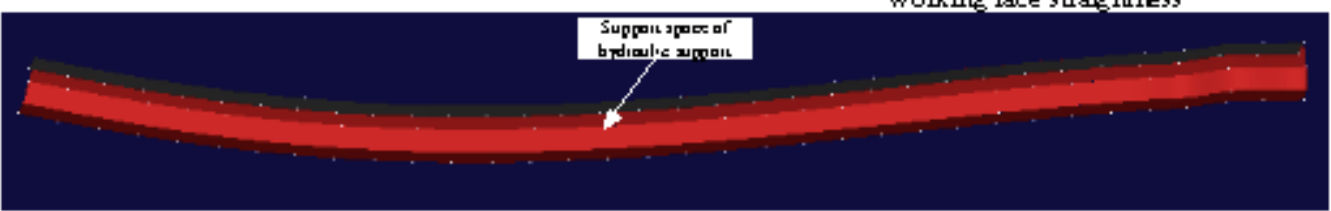

(d)Equipment operation space extraction

Figure 12

Evaluation method

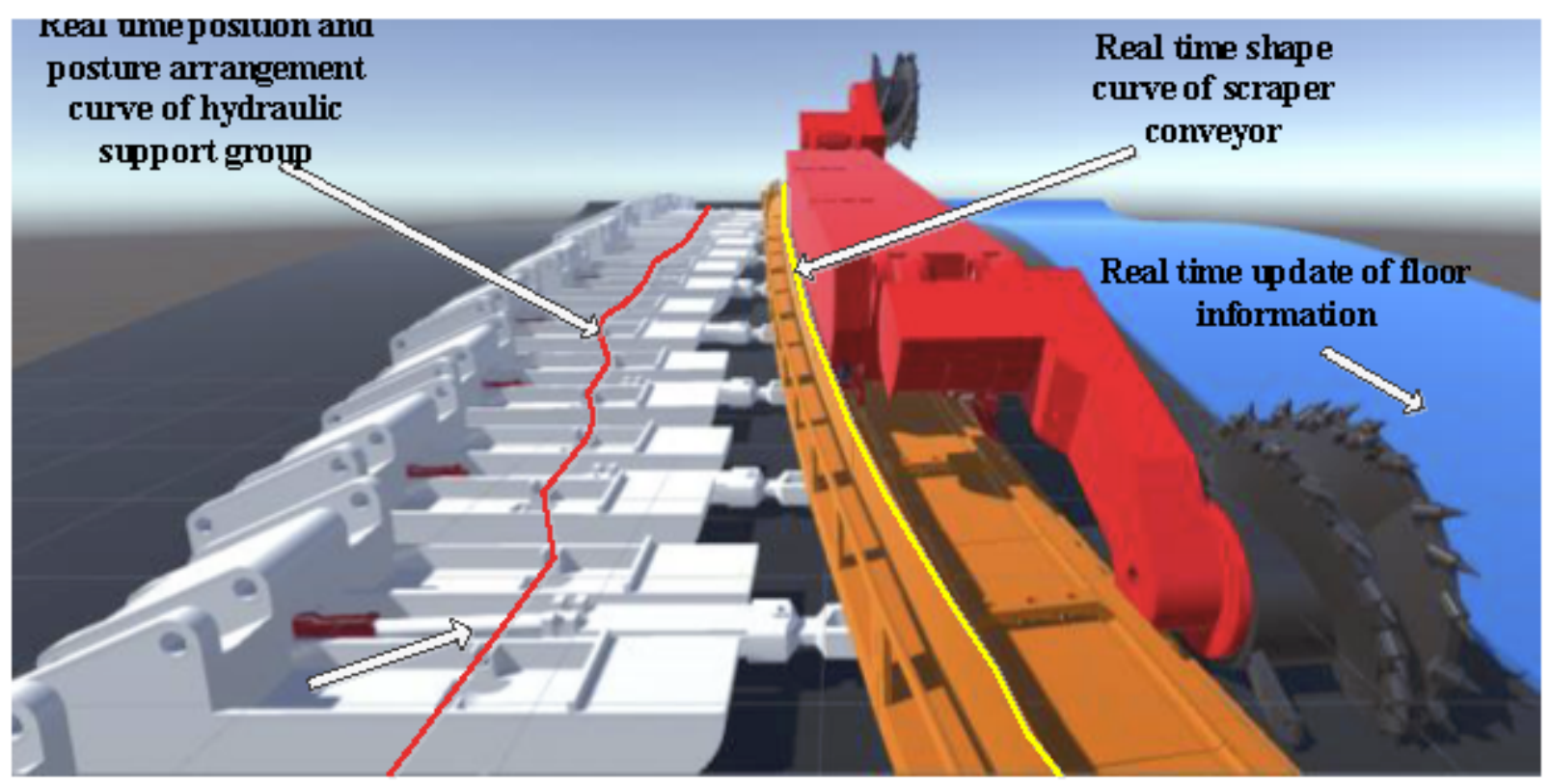

Figure 13

Straightness information extraction of scraper conveyor and hydraulic support group 


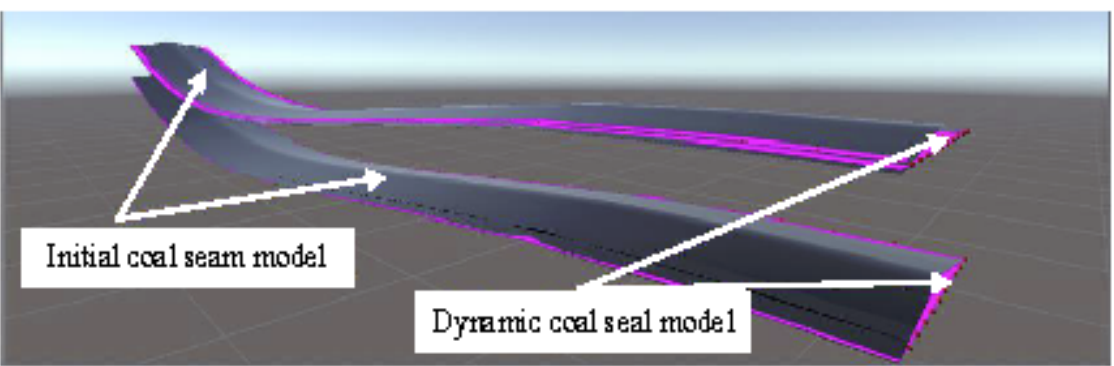

(a) Memory cutting scheme of shearer

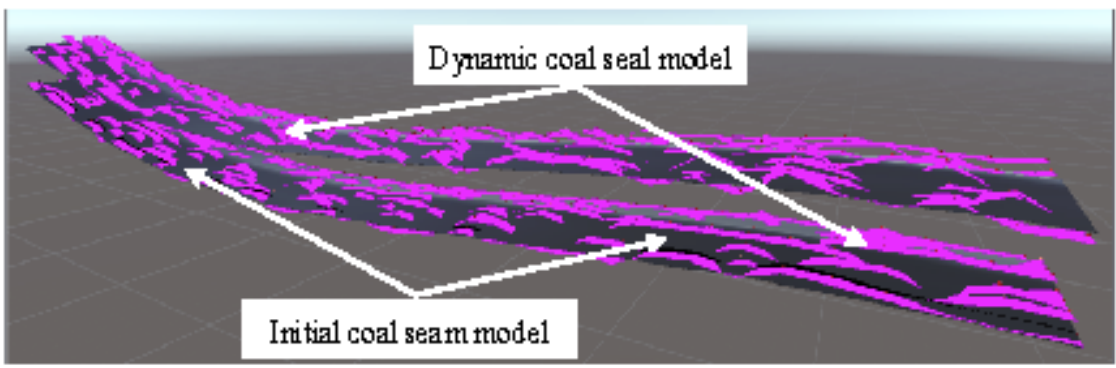

(b) Memory cutting scheme of s hearerwith manual intervention

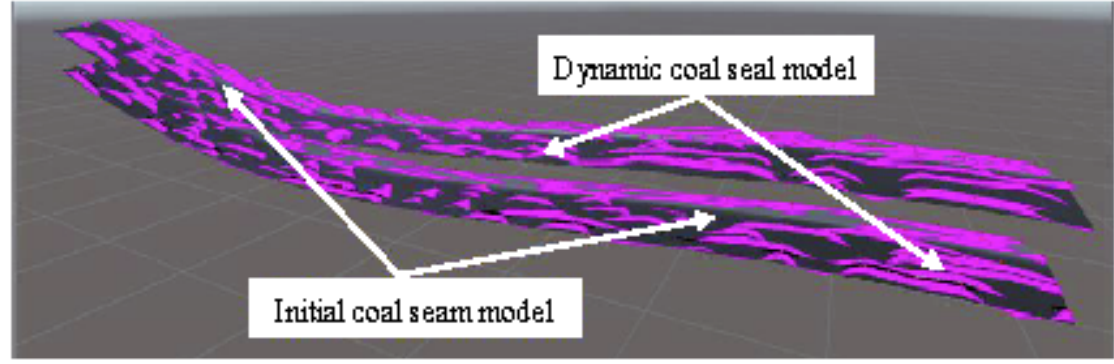

(c) The scheme of self cutting of shearer

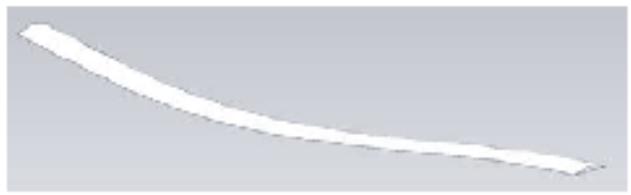

Coal retention

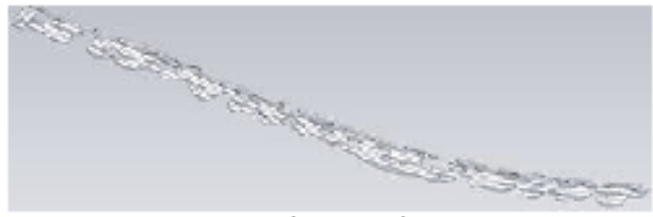

Coal retention

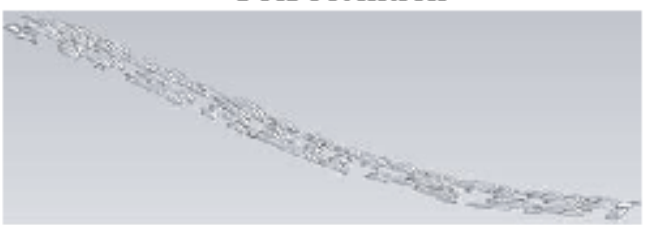

Rock cutting quantity

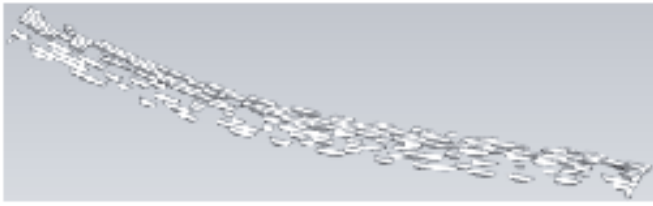

Coal retention

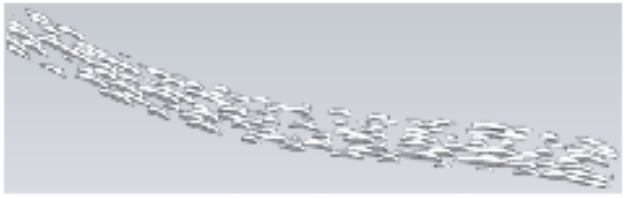

Rock cutting quantity

\section{Figure 14}

Three dimensional schematic diagram of workspace of fully mechanized mining equipment with three cutting schemes 